\title{
Modeling of carbon nanotube Schottky barrier reduction for holes in air
}

\author{
Toshishige Yamada ${ }^{\text {a) }}$ \\ NASA Ames research Center, M/S 229-1, Moffett Field, CA 94035-1000, USA
}

\begin{abstract}
A model is proposed for the previously reported lower Schottky barrier for holes $\Phi_{B h}$ in air than in vacuum at a metallic electrode - semiconducting carbon nanotube (CNT) junction. We consider that there is a transition region between the electrode and the CNT, and an appreciable potential can drop there. The role of the oxidation is to increase this potential drop with negatively charged oxygen molecules on the CNT, leading to lower $\Phi_{\mathrm{Bh}}$ after oxidation. The mechanism prevails in both $p$ - and $n$-CNTs, and the model consistently explains the key experimental findings.
\end{abstract}

\section{INTRODUCTION}

The effects of carbon nanotube (CNT) oxidation have been studied in the thermopower experiments and it has been found that the thermoelectric power coefficient was negative in vacuum and positive in air [1]. In fact, the thermoelectric power coefficient indicates how a bulk internal electric field is created in order to counterbalance the bulk carrier flow due to thermal gradient, and characterizes the bulk properties [2]. The oxidation changes the bulk CNT properties.

Recently, the oxidation effects have been studied using CNT field-effect transistors (FETs) [3], and it has been shown that the contact properties at the electrode and the CNT are modified in oxidation. In these studies, $g_{d}$ was observed to rise slowly at a negative gate voltage $V_{G}\left(V_{G 1}\right)$ and rapidly at a positive $V_{G}\left(V_{G 2}\right)$ in vacuum, while in air, the $g_{d}$ asymmetry flipped, i.e., $g_{d}$ rose rapidly at a negative $V_{G}\left(V_{G 3}\right)$ and slowly at a positive $V_{G}\left(V_{G 4}\right)$. They explained this in terms of the contact property change of the electrode and the CNT: at $V_{G}=0$, the Schottky barrier for holes $\left(\Phi_{B h}\right)$ was high and that for electrons $\left(\Phi_{\mathrm{Be}}\right)$ was low in vacuum while $\Phi_{\mathrm{Bh}}$ was low and $\Phi_{\mathrm{Be}}$ was high in air. Then, $g_{d}$ rose slowly at $V_{G 1}$ since the holes started to tunnel through high $\Phi_{\mathrm{Bh}}$, and $g_{d}$ rose rapidly at $V_{G 2}$ since electrons started to tunnel through low $\Phi_{\mathrm{Be}}$. Similarly, $g_{d}$ rose rapidly at $V_{G 3}$ because of low $\Phi_{\mathrm{Bh}}$ and $g_{d}$ rose slowly at $V_{G 4}$ because of high $\Phi_{\mathrm{Be}}$. In this $g_{d^{-}} V_{G}$ experiment, the contact property modulation of the CNT in oxidation was much more influential than the bulk property change, and the experimental findings were consistently explained through the Schottky barrier modulation.

However, the standard band-alignment theory [4,5] does not explain this Schottky barrier modulation. In this theory, we need to consider the system as a junction of an oxidized metallic electrode surface and a free pure CNT, or of a free pure

metallic electrode and an oxidized CNT. Usually, a gold surface is inert to oxidation, and the first picture is not appropriate. The second picture turns out to give a qualitatively wrong result as discussed below. Generally, a negative charge at the material surface will increase the surface dipole and further confine the electrons within the material [6]. Electronegative oxygen molecules are negatively charged, and the CNT electron affinity $\phi_{\mathrm{s}}{ }^{\prime}$ in air should increase from $\phi_{\mathrm{s}}$ in vacuum such that $\dot{\phi}_{\mathrm{s}}{ }^{\prime}>\phi_{\mathrm{s}}$ (throughout this article, we use a convention that a prime indicates a quantity in air).

(a) vacuum

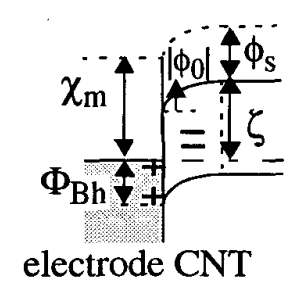

(b) air

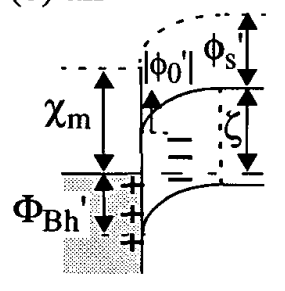

$\begin{array}{ll}\text { (c) vacuum } & \text { (d) air }\end{array}$

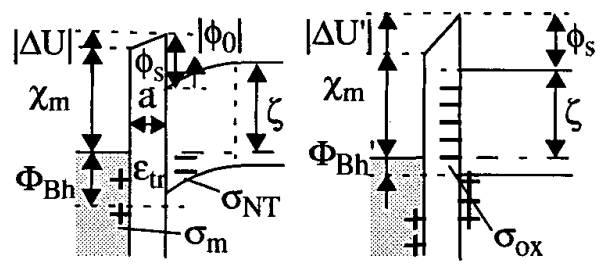

FIG. 1. Band structures for metallic electrode - semiconducting CNT Schottky junction (a) in vacuum and (b) in air in the band-alignment theory with an intimate contact, resulting in the incorrect hole Schottky barrier $\left(\Phi_{\mathrm{Bh}}\right)$ relation, $\Phi_{\mathrm{Bh}}<\Phi_{\mathrm{Bh}}$; Band structures (c) in vacuum and (d) in air in our model, with a potential drop $\Delta U$ in the transition region, resulting in the correct relation of $\Phi_{\mathrm{Bh}}>\Phi_{\mathrm{Bh}} \cdot a$ is the transition region width, $\varepsilon_{\mathrm{tr}}$ is the dielectric constant, $\phi_{\mathrm{s}}$ is the CNT electron affinity, $\zeta$ is the CNT Fermi level, $\chi_{\mathrm{m}}$ is the metallic work function, and $\sigma_{\mathrm{ox}}$ is the negative charge due to oxygen molecules.

Figures 1(a)-1(d) represent band structures across the electrode-CNT junction. The CNT conduction and valence band edges are shown with the Fermi level $\zeta$, and $\chi_{m}$ is a work function of the metallic electrode. According to the standard bandalignment theory, we match the tops of $\chi_{m}$ and $\phi_{s}$ at the inter- 
face as shown in Figs. 1(a) and 1(b). Then, $\Phi_{\mathrm{Bh}}$ in vacuum and $\Phi_{\mathrm{Bh}}$ ' in air are expressed, with $E_{G}$ the CNT band gap, by

$$
\begin{aligned}
& \Phi_{\mathrm{Bh}}=E_{G}+\phi_{\mathrm{s}}-\chi_{\mathrm{m}}, \\
& \Phi_{\mathrm{Bh}}{ }^{\prime}=E_{G}+\phi_{\mathrm{s}}{ }^{\prime}-\chi_{\mathrm{m}} .
\end{aligned}
$$

Thus, $\Phi_{\mathrm{Bh}}<\Phi_{\mathrm{Bh}}{ }^{\prime}$ because $\phi_{\mathrm{s}}<\phi_{\mathrm{s}}{ }^{\prime}$, but this contradicts the experimental observations. In the band-alignment theory, all the oxidation effects are represented in the modulated work function modulation $\phi_{s}{ }^{\prime}$. It is clear that the band-alignment theory does not explain the experiment consistently.

We will propose a model to overcome this difficulty, and explain the Schottky barrier modulation consistently. In our model, we will consider the electrostatic charge balance [7] inside the Schottky junction, while this degree of freedom is not incorporated in the traditional concept of "intimate Schottky junction" in the band-alignment theory.

\section{SCHOTTKY BARRIER MODULATION MECHANISM}

We consider here that the Schottky junction has a sandwich structure, a gold bulk electrode, gold clusters at the electrode surface, charged oxygen molecules, and the CNT surface. The region between the bulk electrode and the CNT is called the "transition region." In fact, a deposited gold surface is rough on the order of $10^{-1} \mu \mathrm{m}$ [8], and oxygen molecules can find a spatial gap (at least in the length direction) to move into or out of the transition region. Moreover, the electrode surface facing the CNT is expected to be microscopically (on the order of several Angstroms) rough since during the electrode deposition process, gold atoms are attracted by the CNT [9] and tend to form clusters. Although the microscopically flat gold surface is known to be inert to oxygen molecules, the clusters on the gold surface can chemisorb oxygen molecules on their tops and form $\mathrm{O}_{2}{ }^{-1} / \mathrm{Au}_{n} / \mathrm{Au}(111)$ with an $n$-dependent binding energy ranging from $\sim 0.45 \mathrm{eV}$ to $\sim 0.96 \mathrm{eV}$ [10]. Thus, the existence of the CNT is critical for the cluster formation and the oxygen chemisorption on the gold electrode surface. There are also discussions about the nature of interaction between the oxygen molecules and the CNT. Some predicted physisorption with negligible charge transfer [11], while others predicted chemisorption and estimated the transferred charge to be -0.1 with a binding energy of $\sim 0.25 \mathrm{eV}$ [12]. In either case, the oxygen molecules dominantly interact with the gold surface with clusters and are negatively charged by -1 , regardless of whether the electrode and the CNT are in the open or closed circuit condition.

The key issue is that a potential drop $\Delta U$ in the transition region is modified in oxidation. As will be shown below, $|\Delta U|$ turns out to be small in vacuum and large in air. This will, in fact, lead to the correct Schottky barrier behavior consistent with the experiments in Ref. 3. Depending on whether $|\Delta U|$ is small in vacuum or large in air, the system has band diagrams as in Figs.1(c) and 1(d). Thus, we have $\Phi_{\mathrm{Bh}}$ in vacuum and $\Phi_{\mathrm{Bh}}{ }^{\prime}$ in air

$$
\begin{aligned}
& \Phi_{\mathrm{Bh}}=\phi_{\mathrm{s}}+E_{G}-\chi_{\mathrm{m}}-|\Delta U| \\
& \Phi_{\mathrm{Bh}}=\phi_{\mathrm{s}}+E_{G}-\chi_{\mathrm{m}}-|\Delta U| .
\end{aligned}
$$

This time, $|\Delta U|<\left|\Delta U^{\prime}\right|$ and $\Phi_{\mathrm{Bh}}>\Phi_{\mathrm{Bh}}$, which is consistent with the experiments. $\chi_{\mathrm{m}}$ is a work function for the free pure metallic surface and $\phi_{\mathrm{s}}$ is an electron affinity for free pure CNT surface. We use the free bulk material parameters $\phi_{s}$ and $\chi_{m}$ in (3) and (4), and all the oxidation effects are included in term $\Delta U$.

The difference between the standard band-alignment theory [4,5] and our model is well represented in the band diagrams: $\Delta U$ is absent in the former as in Figs. 1(a) and 1(b) while $\Delta U$ is present and is significantly modified in oxidation in the latter as in Figs. 1(c) and 1(d). In the band-alignment theory, $\Phi_{\mathrm{Bh}}$ is determined by the bulk properties only, through $\phi_{\mathrm{s}}$ and $\chi_{\mathrm{m}}$. In our model, $\boldsymbol{\Phi}_{\mathrm{Bh}}$ is determined by the potential drop $\Delta U$ in the transition region reflecting the contact properties, in addition to the bulk properties $\phi_{\mathrm{s}}$ and $\chi_{\mathrm{m}}$.

Now we will study how $\Delta U$ is modulated in oxidation in our model. The negative oxygen charge $\sigma_{\mathrm{ox}}$ per unit area is independent of the Fermi energy $\zeta$, and is constant. There are a charge $\sigma_{\mathrm{m}}$ per unit area on the metallic electrode surface and a charge $\sigma_{\mathrm{NT}}$ per unit area within the CNT in the depletion or accumulation mode. The charge neutrality relation requires

$$
\sigma_{\mathrm{m}}+\sigma_{\mathrm{ox}}+\sigma_{\mathrm{NT}}=0
$$

and this determines how $\sigma_{\mathrm{m}}$ appears in the electrode and $\sigma_{\mathrm{NT}}$ appears in the CNT to neutralize $\sigma_{\mathrm{ox}}$. The charge neutrality condition can be split into two equations,

$$
\begin{aligned}
& \sigma=-\sigma_{\mathrm{m}}\left(\phi_{0}\right)=\Delta U \varepsilon_{\mathrm{tt}} \mathrm{J} e a \\
& \sigma=\sigma_{\mathrm{NT}}\left(\phi_{0}\right)+\sigma_{\mathrm{ox}}
\end{aligned}
$$

where $\varepsilon_{\mathrm{tr}}$ is an effective dielectric constant in the transition region, $a$ is a width of the transition region, and $e$ is the unit charge. Once specifying the system including the oxidation level, we know the functional forms $\sigma=-\sigma_{\mathrm{m}}\left(\phi_{0}\right)$ and $\sigma=$ $\sigma_{\mathrm{NT}}\left(\phi_{0}\right)+\sigma_{\mathrm{ox}}$ with a given value of $\sigma_{\mathrm{ox}}$. The intersection of (5a) and (5b) defines $\phi_{0}$ and $\sigma . \sigma$ determines $\Delta U$ through (5a). Then, we calculate $\Phi_{\mathrm{Bh}}$ by (3). The gate voltage $V_{G}$ appears as a modulation of the Fermi level $\zeta$ [13], and determines whether the CNT is $p$-type (large negative $V_{G}$ ) or $n$-type (large positive $V_{G}$ ). The details of the model will be presented elsewhere.

\section{FOUR SCHOTTKY BARRIER BEHAVIORS}

We have found that depending on whether the CNT is $p$ type or $n$-type, and on whether the metallic work function $\chi_{\mathrm{m}}$ is smaller or larger than the CNT work function $\phi_{\mathrm{s}}+\zeta$, there are four different behaviors. They are summarized as follows:

\section{(1) $p$-CNT when $\chi_{\mathrm{m}}<\phi_{\mathrm{s}}+\zeta$}

In vacuum, $\sigma=\sigma_{\mathrm{NT}}\left(\phi_{0}\right)+\sigma_{\mathrm{ox}}$ with $\sigma_{\mathrm{ox}}=0$ has an intersection Avac with a large negative (downward) band bending 
$\phi_{0}$ in the inversion mode and a small negative $\sigma$. Because of (5a), the small negative $\sigma$ corresponds to a small negative (right up, left down) $\Delta U$. The band structure is shown in Fig. 2(a). As the oxidation progresses and $\sigma_{\mathrm{ox}}$ becomes more negative, and $\sigma=\sigma_{\mathrm{NT}}\left(\phi_{0}\right)+\sigma_{\mathrm{ox}}$ shifts downward. The intersection Aox in the strong oxidation limit has a negligible $\phi_{0} \sim 0$ (almost flat band) in the accumulation mode and a large negative $\sigma$ corresponding to a large negative (right up) $\Delta U$. The band structure is shown in Fig. 2(b). It is clearly seen that $\Phi_{\mathrm{Bh}}$ decreases appreciably in oxidation.

\section{(2) p-CNT when $\chi_{\mathrm{m}}>\phi_{\mathrm{s}}+\zeta$}

The intersection occurs only at the accumulation portion of $\sigma=\sigma_{\mathrm{NT}}\left(\phi_{0}\right)+\sigma_{\mathrm{ox}}$ regardless of $\sigma_{\mathrm{ox}}$ and has a negligible $\phi_{0}$ $\sim 0$ (almost flat band) and a large positive $\sigma$ corresponding to a large positive (right down) $\Delta U$. The Schottky barrier modulation is negligible (band structure not shown).

\section{(3) $n$-CNT when $\chi_{m}>\phi_{s}+\zeta$}

In vacuum, $\sigma=\sigma_{\mathrm{NT}}\left(\phi_{0}\right)+\sigma_{\mathrm{ox}}$ with $\sigma_{\mathrm{ox}}=0$ has an intersection Bvac with a small positive (upward) $\phi_{0}$ and a small positive $\sigma$ corresponding to a small positive (right down) $\Delta U$. The band structure is shown in Fig. 2(c). As the oxidation progresses, $\sigma=\sigma_{\mathrm{NT}}\left(\phi_{0}\right)+\sigma_{\mathrm{ox}}$ shifts downward. The $n$-CNT is locked to the inversion mode regardless of $\sigma_{\mathrm{ox}}$. The intersection Box in the strong oxidation limit has a large positive (upward) $\phi_{0}$ and a large negative $\sigma$ corresponding to a large negative (right up) $\Delta U$. The band structure is shown in Figs. 2(d). It is clearly seen that $\Phi_{\mathrm{Be}}$ increases appreciably in oxidation.

\section{(4) $n$-CNT when $\chi_{\mathrm{m}}<\phi_{\mathrm{s}}+\zeta$}

In the weak oxidation $0>\sigma_{\mathrm{ox}}>\left[\chi_{\mathrm{m}}-\left(\phi_{\mathrm{s}}+\zeta\right)\right] \varepsilon_{\mathrm{tr}} / e a$, the Schottky barrier modulation is negligible, but in the strong oxidation $\left[\chi_{\mathrm{m}}-\left(\phi_{\mathrm{s}}+\zeta\right)\right] \varepsilon_{\mathrm{tr}} / e a>\sigma_{\mathrm{ox}}$, a finite Schottky barrier modulation is expected (band structure not shown).

\section{DISCUSSIONS}

The Schottky barrier modulation due to oxidation was significant for both negative and positive $V_{G}$ bias conditions in Ref. 3. Thus, we can exclude case (2) and case (4). The CNT was $p$-type at the negative $V_{G}$ onset at $V_{G I}$ in vacuum and at $V_{G 3}$ in air, and this should correspond to case (1). Then, the condition $\chi_{\mathrm{m}}<\phi_{\mathrm{s}}+\zeta$ had to be satisfied. Similarly, the CNT was $n$-type at the positive $V_{G}$ onset at $V_{G 2}$ in vacuum and at $V_{G 4}$ in air, and this should correspond to case (3). Then, the condition $\chi_{\mathrm{m}}>\phi_{\mathrm{s}}+\zeta$ had to be satisfied. Therefore, we had $\chi_{\mathrm{m}}$ $<\phi_{\mathrm{s}}+\zeta$ for a negative $V_{G}$ and $\chi_{\mathrm{m}}>\phi_{\mathrm{s}}+\zeta$ for a positive $V_{G}$. This is possible when the work function difference at $V_{G}=0$, $\left|\chi_{\mathrm{m}}-\left(\phi_{\mathrm{s}}+\zeta_{0}\right)\right|$, was small enough compared to the band gap $E_{G}$ in the gold-CNT system. We believe that this was in fact the case. Because of an obvious relation $\Phi_{\mathrm{Bh}}+\Phi_{\mathrm{Be}}=\Phi_{\mathrm{Bh}}{ }^{\prime}+\Phi_{\mathrm{Be}}{ }^{\prime}$ $=E_{G}$, a large positive band bending $\phi_{0}$ at $V_{G 1}$ (Avac) should occur with a small negative band bending $\phi_{0}$ at $V_{G 2}$ (Bvac) in vacuum. In air, a small negative band bending $\phi_{0}$ at $V_{G 3}$ (Aox) should occur with a large positive band bending $\phi_{0}$ at $V_{G 4}$ (Box). We can clearly see this trend in our results. The experimentally observed Schottky barrier modulation in Ref. 3 is consistently explained: Fig. 2(a) corresponds to $V_{G I}$; Fig. 2(c) corresponds to $V_{G 2}$; Fig. 2(b) corresponds to $V_{G 3}$; Fig. 2(d) corresponds to $V_{G 4}$.
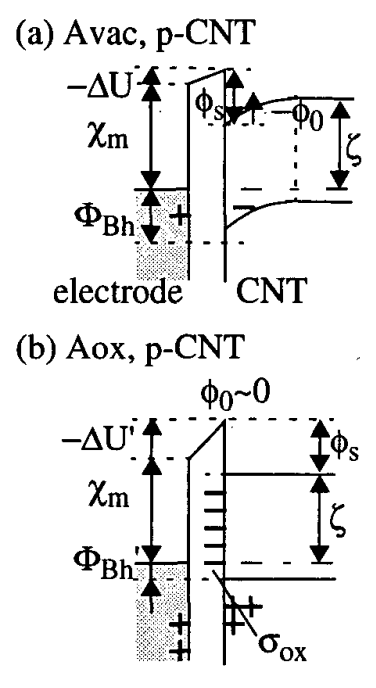

(c) Bvac, $\mathrm{n}-\mathrm{CNT}$

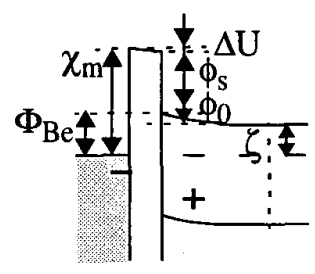

(d) Box, n-CNT

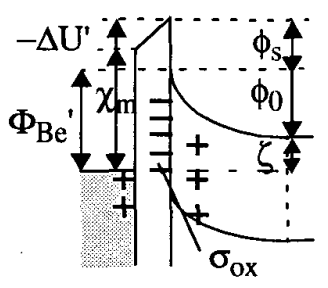

FIG. 2. Band structures are shown for four typical Schottky barrier behaviors represented by the intersections: (a) Avac for $p$-CNT in vacuum and (b) Aox for $p$-CNT in air, demonstrating the correct relation $\Phi_{\mathrm{Bh}}>\Phi_{\mathrm{Bh}}{ }^{\prime}$; (c) Bvac for $n$-CNT in vacuum and (d) Box for $n$-CNT in air, demonstrating the correct relation $\Phi_{\mathrm{Be}}<\Phi_{\mathrm{Be}}$ '

We next estimate the relevant numerical values. In order to define the negative oxygen charge density, we project the charged oxygen molecules to a gold (111) plane. If $\Gamma_{\mathrm{ox}} \%$ of the gold plane is covered with oxygen molecules, corresponding to $\Gamma_{\mathrm{ox}}$ oxygen molecules per one hundred gold atoms, $\sigma_{\mathrm{ox}}$ ' $e=-1.86 \times 10^{13} \Gamma_{\mathrm{ox}} \mathrm{cm}^{-2}$. Then, the contribution of $\sigma_{\mathrm{ox}}$ upon $\Delta U$ is estimated to be $0.251 \times a(\AA) \Gamma_{\mathrm{ox}}(\%) / \varepsilon_{\mathrm{tr}} \mathrm{eV}$. According to an adsorption experiment of the gold free cluster-oxygen molecule system at room temperature, the reaction completion is quite high, larger than $80 \%$ [14]. We can expect a similarly high completion ratio for the supported clusters on the $\mathrm{Au}(111)$ surface facing the CNT, which is of our interest. Thus, many supported clusters have negatively charged oxygen molecules on their tops. There are no direct data to estimate the supported cluster density in the context of the CNT. Using the projected oxygen molecule density onto the Au (111) surface, $\Gamma_{\text {ox }} \sim 10^{-1}$. $10^{0} \%$ would not be impossible. A gold cluster height $b$ with an oxygen molecule is $2-4 \AA$ [10]. Thus, we can expect the transition region width to be $a \sim 3-5 \AA$. A gas, including oxygen, has a dielectric constant close to unity. The gold clusters effectively increases the dielectric constant by $1 /[1-b / a]$ and this needs to be multiplied by an appropriate weighting factor as a function of $\Gamma_{\mathrm{ox}}$ reflecting the partial coverage with clusters. Thus, we 
expect $\varepsilon_{\mathrm{tr}} \sim 10^{0}$. Having $\Gamma_{\mathrm{ox}} \sim 10^{-1}-10^{0} \%, a \sim 3-5 \AA$, and $\varepsilon_{\mathrm{tr}} \sim$ $10^{0}$ in mind, we find that $a \Gamma / \varepsilon_{\text {tr }}$ is on the order of unity and the contribution of $\sigma_{\mathrm{ox}}$ to $\Delta U$ is a few tenths of an electron volt. Considering $E_{G} \sim 0.5 \mathrm{eV}$ for a familiar semiconducting $(17,0)$ CNT [15], the present model will produce the expected oxidation effects.

\section{ACKNOWLEDGMENT}

The author gratefully acknowledges Alessandra Ricca for providing preprints and discussions, and M. Meyyappan for discussions and a critical reading of the manuscript.

\section{REFERENCES}

a) CSC, Electronic address: yamada@nas.nasa.gov

[1] G. U. Sumanasekera, C. K. W. Adu, S. Fang, and P. C. Eklund, "Effects of gas adsorption and collisions on electrical transport in single-walled carbon nanotubes", Phys. Rev. Lett. Vol. 85, pp.1096-1099 (2000); K. Bradley, S.-H. Jhi, P. G. Collins, J. H one, M. L. Cohen, S. G. Louie, and A. Zettl, "Is the intrinsic thermoelectric power of carbon nanotube positive?", Phys. Rev. Lett. Vol. 85, pp. $4361-4364$ (2000).

[2] H. Takahashi, Electromagnetism. Tokyo, Japan: Shokabo, 1959; R. Kubo, Statistical Mechanics. New York, NY: North-Holland, 1965.

[3] V. Derycke, R. Martel, J. Appenzeller, and Ph. Avouris, "Carbon nanotube inter- and intramolecular logic gates", Nano Lett. Vol. 1, pp.453 (2001); R. Martel, V. Derycke. J. Appenzeller, K. K. Chan, J. Tersoff, and $\mathrm{Ph}$. Avouris, "Ambipolar electrical transport in semiconducting singlewall carbon nanotubes", Phys. Rev. Lett. Vol. 87, pp.256805 (2001); S. Heinze, J. Tersoff, R. Martel, V. Derkcke, J. Appenzeller, and Ph. Avouris, "Carbon nanotubes as Schottky barrier transistors", Phys. Rev. Lett. Vol. 89, pp.106801 (2002); J. Appenzeller, J. Koch, V. Derycke, R. Martel, S. Wind, and Ph. Avouris, "Field-modulated transport in carbon nanotube transistors", Phys. Rev. Lett. Vol. 89, pp.126801 (2002).

[4] S. M. Sze, Physics of Semiconductor Devices, 2nd ed. New York, NY: Wiley, 1981

[5] T. Yamada, "Modeling of electronic transport in scanning tunneling microscope4 tip - carbon nanotube systems", Appl. Phys. Lett. Vol. 78, pp.1739-1741 (2001).

[6] G. Attard and C. Barnes, Surfaces, Oxford Chemistry Primes. New York, NY: Oxford, 1998.

[7] J. Bardeen, "Surface states and rectification at a metal semi-conductor contact", Phy. Rev. Vol. 71, pp.717-727 (1947); W. H. Brattain and J. Bardeen, "Surface properties of germanium", Bell Syst. Tech. J. Vol. 32, pp.1-41 (1952).

[8] M. Aguilar, E. Anguiano, J. A. Aznarez, and J. L. Sacedon, "Rough growth fronts of evaporated gold films, compared with self-affine and mound growth models", Surf. Sci. Vol. 482-485, pp.935-939 (2001).

[9] J. Liu, A. G. Rinzler, H. Dai, J. H. Hafner, R. K. Bradley, P. J. Boul, A. Lu, T. Iverson, K. Shelimov, C. B. Huffman, F. Rodrigez-Marcias, Y.-S. Shon, T. R. Lee, D. T. Colbert, R. E. Smalley, "Fullerene pipes", Science, Vol. 280, 1253-1256 (1998). L. Liu, T. Wang, J. Li, Z.-X. Guo, L. Dai, D. Zhang, and D. Zhu, "Self-assembly of gold nanoparticles to carbon nanotubes using a thiol-terminated pyrene as interlinker," Chem Phys. Lett. Vol. 367, pp.747-752 (2003).

[10] G. Mills, M. S. Gordon, and H. Metiu, "Oxygen adsorption on Au clusters and a rough $\mathrm{Au}(111)$ surface: the role of surface flatness, electron confinement, excess electrons, and band gap", J. Chem. Phys. Vol. 118 , pp.4198-4205 (2003).

[11] A. Ricca, preprint. See also, A. Ricca and J. A. Dorocco, "Interaction of $\mathrm{O}_{2}$ with a $(9,0)$ carbon nanotube", Chem. Phys. Lett. Vol. 367, pp.217223 (2002).

[12] S.-H. Jhi, S. G. Louie, and M. L. Cohen, "Electronic properties of oxidized carbon nanotubes", Phys. Rev. Lett. Vol. 85, pp.1710-1703 (2000).

[13] T. Yamada, "Modeling of kink-shaped carbon-nanotube Schottky diode with gate bias modulation", Appl. Phys. Lett. Vol. 80, pp.4027-4029 (2002).
[14] B. E. Salisbury, W. T. Wallace, and R. L. Whetten, "Low-temperature activation of molecular oxygen by gold clusters: a stoichiometric process correlated to electron affinity", Chem. Phys. Vol. 262, pp.131-141 (2000).

[15] M. S. Dresselhaus, G. Dresselhaus, and P. C. Eklund, Science of Fullerenes and Carbon Nanotubes. San Diego, CA: Academic, 1996. 